\title{
ON THE EXTENSION OF A FUNCTIONAL INEQUALITY OF S. BERNSTEIN TO NON-ANALYTIC FUNCTIONS ${ }^{1}$
}

\author{
R. J. DUFFIN AND A. C. SCHAEFFER
}

We wish to demonstrate here the following elementary inequality of the differential calculus. If a function ${ }^{2}$ satisfies the conditions $\{f(x)\}^{2} \leqq 1$ and $\left\{f^{(n)}(x)\right\}^{2}+\left\{f^{(n-1)}(x)\right\}^{2} \leqq 1$ for all $x$ and for some positive integer $n$, then the latter inequality is valid also when $n$ is replaced by any smaller positive integer.

That such an inequality might be true is suggested by the validity of a similar but more specialized inequality concerning trigonometric polynomials. ${ }^{3}$ Thus, if $P(x)=\sum_{0}^{N}\left\{a_{\nu} \cos (\nu x / N)+b_{\nu} \sin (\nu x / N)\right\}$ and if $\{P(x)\}^{2} \leqq 1$ for all $x$, it has been proved that $\left\{P^{(k)}(x)\right\}^{2}+\left\{P^{(k-1)}(x)\right\}^{2}$ $\leqq 1,(k=1,2,3, \cdots)$. This theorem, a refinement of a theorem of S. Bernstein, has been proved by several different methods, ${ }^{4}$ and generalizations have been given which prove that the inequality is true for a wider class of analytic functions. It will be shown here that this theorem is a rapid deduction from the elementary inequality given above. Moreover, this method of proof serves to distinguish those features of Bernstein's theorem arising from the characteristic properties of trigonometric polynomials from those which are merely properties of the differential coefficient.

The second part of this paper is concerned with finding the functions which cause the inequality to become an equality at some point. For example, if $\left\{f^{(3)}(x)\right\}^{2}+\left\{f^{(2)}(x)\right\}^{2} \leqq 1$ and $\{f(x)\}^{2} \leqq 1$, we find that the equality $f^{\prime}(0)=1$ necessitates that $f(x) \equiv \sin x$. It is to be noted

${ }^{1}$ Presented to the Society, September 6, 1938, under the title Extension of a theorem of S. Bernstein to non-analytic functions.

${ }^{2}$ In stating that an inequality involving $f^{(n)}(x)$ is satisfied in an interval we imply that the derivatives of all orders up to and including the $n$th exist in the same interval. All functions and constants appearing in this paper are considered real unless the contrary is explicitly stated.

${ }^{3}$ In formulating such a comparison, we have taken a hint from a paper by Oystein Ore, On functions with bounded derivatives, Transactions of this Society, vol. 43 (1938), pp. 321-326. Ore introduces an "extension principle" which indicates how certain theorems concerning ordinary algebraic polynomials may be modified in order to be applicable to arbitrary functions.

${ }^{4}$ G. Szegö, Schriften der Königsberger gelehrten Gesellschaft, 5th year, no. 4 (1928), pp. 59-70. J. van der Corput and G. Schaake, Compositio Mathematica, vol. 2 (1935), pp. 321-361; vol. 3 (1936), p. 128. R. Boas, Transactions of this Society, vol. 40 (1936), pp. 287-308. R. Duffin and A. Schaeffer, this Bulletin, vol. 43 (1937), pp. 554-556; vol. 44 (1938), pp. 236-240. 
that the behavior of this system of differential inequalities is similar to the behavior of a differential equation because the assignment of certain initial values gives rise to a unique solution. It is possible to construct other examples of differential inequalities with this stability property.

The method of proof employs only fundamental notions. Essentially all that is needed is the mean value theorem and Weierstrass' theorem on the maximum of a continuous function.

THEOREM A. If $f(x)$ is a function which for some integer $n$ and for all $x$ satisfies the conditions

$$
\begin{array}{r}
\{f(x)\}^{2} \leqq 1, \\
\left\{f^{(n)}(x)\right\}^{2}+\left\{f^{(n-1)}(x)\right\}^{2} \leqq 1,
\end{array}
$$

then for $k=1,2,3, \cdots, n$ and for all $x$

$$
\left\{f^{(k)}(x)\right\}^{2}+\left\{f^{(k-1)}(x)\right\}^{2} \leqq 1
$$

Proof. Considering first the case $n=2$, we have by hypothesis $\{f(x)\}^{2} \leqq 1$ and $\left\{f^{\prime}(x)\right\}^{2}+\left\{f^{\prime \prime}(x)\right\}^{2} \leqq 1$. It is to be shown that the function $\phi(x) \equiv\{f(x)\}^{2}+\left\{f^{\prime}(x)\right\}^{2}$ is not greater than 1. Suppose, on the contrary, that at some point $x_{1}, \phi\left(x_{1}\right)=1+2 \delta, \delta>0$. If $x$ is any point to the right of $x_{1}$, then by the mean value theorem a point $x_{2}$ exists such that

$$
f^{\prime}\left(x_{2}\right)=\left\{f(x)-f\left(x_{1}\right)\right\} /\left(x-x_{1}\right), \quad x_{1}<x_{2}<x .
$$

Taking $x-x_{1}=2 /(\delta)^{1 / 2}$, we see that $\left|f^{\prime}\left(x_{2}\right)\right| \leqq(\delta)^{1 / 2}$. Hence $\phi\left(x_{2}\right) \leqq 1$ $+\left\{f^{\prime}\left(x_{2}\right)\right\}^{2} \leqq 1+\delta$. The same method is available to show that there is a point to the left of $x_{1}$ where $\phi(x) \leqq 1+\delta$. Thus, $\phi(x)$ is greater at $x_{1}$ than it is at certain points to the left and to the right; it must, therefore, have a maximum in the interior of the interval defined by these points. At this maximum point, say $x_{0}$, we have $\phi\left(x_{0}\right) \geqq 1+2 \delta$; and the derivative is equal to zero:

$$
2 f^{\prime}\left(x_{0}\right)\left\{f\left(x_{0}\right)+f^{\prime \prime}\left(x_{0}\right)\right\}=0 .
$$

But, if the first factor is zero, then $\phi\left(x_{0}\right)=\left\{f\left(x_{0}\right)\right\}^{2} \leqq 1$, while if the second factor is zero, then

$$
\phi\left(x_{0}\right)=\left\{-f^{\prime \prime}\left(x_{0}\right)\right\}^{2}+\left\{f^{\prime}\left(x_{0}\right)\right\}^{2} \leqq 1 .
$$

This contradiction proves the theorem for $n=2$.

For $n$ greater than 2 we proceed by induction. Thus, suppose the theorem is true for $n=m-1$ and consider the case $n=m$. First, in order to show that $f^{\prime}(x)$ is bounded uniformly in $x$, we obtain the 
crude estimate $\left|f^{\prime}\left(x_{0}\right)\right| \leqq 5$ for an arbitrary point $x_{0}$. By the mean value theorem

$$
f\left(x_{0}+1\right)-f\left(x_{0}\right)=f^{\prime}\left(x_{0}+h\right), \quad 0<h<1 .
$$

Because $f$ is bounded by 1 , this gives, for some $h$ between 0 and 1 ,

$$
\left|f^{\prime}\left(x_{0}+h\right)\right| \leqq 2 \text {. }
$$

Again, by the mean value theorem, if $x$ is any point and $h$ is the same constant appearing in (1), then for some point $x_{1}$

$$
f^{(m-2)}(x+h)-f^{(m-2)}(x)=h f^{(m-1)}\left(x_{1}\right) .
$$

But, by hypothesis $f^{(m-1)}$ is bounded by 1 ; so, using (2), we obtain

$$
\left\{f^{(m-2)}(x+h)-f^{(m-2)}(x)\right\}^{2}+\left\{f^{(m-1)}(x+h)-f^{(m-1)}(x)\right\}^{2} \leqq 9 .
$$

Note that $\{f(x+h)-f(x)\}^{2}<9$ follows from hypothesis (a), so the theorem is applicable to the function $\{f(x+h)-f(x)\} / 3$ in the case $n=m-1$ for which it has been supposed true. As a consequence

$$
\left\{\frac{f^{\prime}(x+h)-f^{\prime}(x)}{3}\right\}^{2}+\left\{\frac{f(x+h)-f(x)}{3}\right\}^{2} \leqq 1 .
$$

In particular the last relation gives for the point $x_{0}$ the inequality

$$
\left|f^{\prime}\left(x_{0}+h\right)-f^{\prime}\left(x_{0}\right)\right| \leqq 3 \text {. }
$$

Combining (1) and (3) we obtain the desired bound $\left|f^{\prime}\left(x_{0}\right)\right| \leqq 5$.

Let $\beta$ be the least upper bound of $\left|f^{\prime}(x)\right|$ for all values of $x$; we have just shown that $\beta$ is finite. If $\beta>1$, the function $\beta^{-1} f^{\prime}(x)$ satisfies the conditions of the theorem in the case $n=m-1$ and consequently

$$
\left\{\beta^{-1} f^{\prime \prime}(x)\right\}^{2}+\left\{\beta^{-1} f^{\prime}(x)\right\}^{2} \leqq 1 .
$$

Let $F(x)=\beta^{-1} f(x)+\gamma\left(1-\beta^{-1}\right)$ where $\gamma=+1$ or -1 and is to be determined later. Because $\{f(x)\}^{2} \leqq 1$, clearly $\{F(x)\}^{2} \leqq 1$; and by (4), $\left\{F^{\prime \prime}(x)\right\}^{2}+\left\{F^{\prime}(x)\right\}^{2} \leqq 1$. Applying the theorem to the function $F(x)$ in the case $n=2$, we have $\left\{F^{\prime}(x)\right\}^{2}+\{F(x)\}^{2} \leqq 1$ or

$$
\left\{\beta^{-1} f^{\prime}(x)\right\}^{2}+\left\{\beta^{-1} f(x)\right\}^{2}+2 \gamma \beta^{-1}\left(1-\beta^{-1}\right) f(x)+\left(1-\beta^{-1}\right)^{2} \leqq 1 .
$$

By the definition of $\beta$ there exist points for which the first term on the left is arbitrarily close to 1 . And for fixed $x$ the term containing $\gamma$ can be made nonnegative since $\gamma$ is either +1 or -1 . Thus, the least upper bound of the left-hand side is not smaller than $1+\left(1-\beta^{-1}\right)^{2}$; and because this can be no larger than 1 , it follows that $\beta \leqq 1$. 
The problem is by the above considerations reduced to the case $\left\{f^{\prime}(x)\right\}^{2} \leqq 1$. Applying the theorem to the function $f^{\prime}(x)$ with $n=m-1$, we have for $k=2,3,4, \cdots, m-1, m$

$$
\left\{f^{(k)}(x)\right\}^{2}+\left\{f^{(k-1)}(x)\right\}^{2} \leqq 1 .
$$

But, now we may apply the theorem to $f(x)$ with $n=2$, and this shows that (c) is true for $k=1$ also. ${ }^{5}$

We wish now to determine the class of functions which make relation (c) of Theorem A an equality at some point and for some value of $k$. The following lemma is of central importance for this investigation.

Lemma. If the function $f(x)$ satisfies in the interval $0 \leqq \alpha \leqq x \leqq \pi / 2$ the conditions

$$
\begin{gathered}
\left\{f^{\prime \prime}(x)\right\}^{2}+\left\{f^{\prime}(x)\right\}^{2} \leqq 1, \\
f(\alpha)=\sin \alpha, \quad f^{\prime}(\alpha)=\cos \alpha, \\
f(\pi / 2) \leqq 1,
\end{gathered}
$$

then in the same interval

$$
f(x) \equiv \sin x .
$$

PROoF. It is convenient to introduce two auxiliary functions $\omega(x)$ and $\psi(x)$, defined by the relations

$$
\begin{aligned}
\omega(x) & =f(x)-\sin x, \\
2 \psi(x) & =1+\left\{\omega^{\prime \prime}(x)\right\}^{2}+\left\{\omega^{\prime}(x)\right\}^{2}-\left\{f^{\prime \prime}(x)\right\}^{2}-\left\{f^{\prime}(x)\right\}^{2} .
\end{aligned}
$$

By condition (b) $\omega(\alpha)=\omega^{\prime}(\alpha)=0$, and by (a) $\psi(x)$ is nonnegative in the interval under consideration. Writing $f(x)=\omega(x)+\sin x$ and substituting in the expression for $2 \psi(x)$ gives

$$
\psi(x)=\sin x \omega^{\prime \prime}(x)-\cos x \omega^{\prime}(x) ;
$$

and if $x \neq 0$, this relation may be put in the form

$$
\frac{d}{d x}\left(\omega^{\prime}(x) / \sin x\right)=\psi(x) / \sin ^{2} x .
$$

5 The following three modifications of Theorem A are of some interest: (1) The assumption that $f^{(n)}(x)$ exists may be replaced by weaker conditions. For example, in condition (b), $\left\{f^{(n)}(x)\right\}^{2}$ may be interpreted as $\lim \sup _{h \rightarrow \pm 0}\left\{\left(f^{(n-1)}(x+h)-f^{(n-1)}(x)\right) / h\right\}^{2}$. (2) The theorem may be stated for a finite interval provided the inequalities (c) are given to be satisfied at the end points of the interval for every value of $k$. (3) The theorem is valid for complex functions $f(x)$ provided $|f(x)| \leqq 1$ and relations of the form $\left\{f^{(R)}(x)\right\}^{2}+\left\{f^{(R-1)}(x)\right\}^{2} \leqq 1$ are replaced by relations of the form $\mid f^{(R)}(x) \cos \alpha$ $+f^{(R-1)}(x) \sin \alpha \mid \leqq 1$ for all real $x$ and $\alpha$. 
Considering first the case $\alpha>0$, we have by the mean value theorem if $0<\alpha<x<\pi / 2$

$$
\omega^{\prime}(x) / \sin x-\omega^{\prime}(\alpha) / \sin \alpha=(x-\alpha) \psi\left(x_{1}\right) / \sin ^{2} x_{1}, \quad \alpha<x_{1}<x .
$$

But, $\omega^{\prime}(\alpha)=0$ and $\psi(x) \geqq 0$; so

$$
\omega^{\prime}(x) \geqq 0, \quad \alpha \leqq x \leqq \pi / 2,
$$

which implies, by the mean value theorem, that $\omega(x)$ is non-decreasing in this interval. Because $\omega$ vanishes at $x=\alpha$ and because, by (c), $\omega$ cannot be greater than zero at $x=\pi / 2$, it follows from (2) that $\omega(x)$ vanishes identically in the interval $(\alpha, \pi / 2)$.

Special attention must be given the case $\alpha=0$. If $x_{0}<x$ are any two points in the interior of the interval $(0, \pi / 2)$, by the mean value theorem the relation (1) gives

$$
\begin{aligned}
\omega^{\prime}(x) / \sin x-\omega^{\prime}\left(x_{0}\right) / \sin x_{0}=\left(x-x_{0}\right) \psi\left(x_{1}\right) / \sin ^{2} x_{1}, \\
0<x_{0}<x_{1}<x<\pi / 2 .
\end{aligned}
$$

Consider the limiting form of this relation as $x_{0}$ approaches zero for fixed $x$. The second term on the left approaches $\omega^{\prime \prime}(0)$, and the expression on the right, being always nonnegative, must have a nonnegative limit. By conditions (a) and (b) it is clear that $f^{\prime \prime}(\alpha)=0$ if $\alpha=0$, and this implies that $\omega^{\prime \prime}(0)=0$. Thus relation (2) is valid also for the case $\alpha=0$, and $\omega(x)$ must vanish identically in the interval $(0, \pi / 2)$; this completes the proof.

THEOREM B. If $f(x)$ is a function which for all $x$ satisfies the conditions

$$
\begin{array}{r}
\{f(x)\}^{2} \leqq 1 \\
\left\{f^{\prime \prime}(x)\right\}^{2}+\left\{f^{\prime}(x)\right\}^{2} \leqq 1,
\end{array}
$$

and if at some point $x_{1}$

$$
\left\{f^{\prime}\left(x_{1}\right)\right\}^{2}+\left\{f\left(x_{1}\right)\right\}^{2}=1
$$

then for some constant $\gamma$

$$
f(x) \equiv \sin (x+\gamma)
$$

in an interval from $x_{1}$ to the nearest point where $|\sin (x+\gamma)|=1$, and in case $f\left(x_{1}\right)=0$ the interval extends a distance $\pi / 2$ on each side of $x_{1}$.

PRoof. There is no loss of generality in supposing $f\left(x_{1}\right) \geqq 0, f^{\prime}\left(x_{1}\right) \geqq 0$ because the general case may be reduced to this by considering the function $\pm f( \pm x)$ for some choice of signs. (If $f\left(x_{1}\right)=0$, the signs can 
be chosen in two ways.) Thus let $f\left(x_{1}\right)=\sin \alpha$ and $f^{\prime}\left(x_{1}\right)=\cos \alpha$, $0 \leqq \alpha \leqq \pi / 2$. Again there is no loss of generality if we assume $x_{1}=\alpha$ since this situation may be realized by a suitable translation of the origin. Application of the preceding lemma completes the proof of Theorem B.

THEOREM C. If $f(x)$ is a function which for all $x$ and for some integer $n$ greater than 2 satisfies the conditions

$$
\begin{aligned}
\{f(x)\}^{2} & \leqq 1, \\
\left\{f^{(n)}(x)\right\}^{2}+\left\{f^{(n-1)}(x)\right\}^{2} & \leqq 1,
\end{aligned}
$$

and if at some point $x_{1}$ and for some integer $k$ in the range $1 \leqq k \leqq n-1$,

$$
\left\{f^{(k)}\left(x_{1}\right)\right\}^{2}+\left\{f^{(k-1)}\left(x_{1}\right)\right\}^{2}=1 \text {, }
$$

then there is a constant $\gamma$ such that for all $x$

$$
f(x) \equiv \sin (x+\gamma)
$$

except possibly if $k=1$ and $\left\{f\left(x_{1}\right)\right\}^{2}=1$.

Proof. We shall tacitly employ Theorem A throughout. First consider the case $k=1$; suppose

$$
\left\{f^{\prime}\left(x_{1}\right)\right\}^{2}+\left\{f\left(x_{1}\right)\right\}^{2}=1, \quad\left\{f\left(x_{1}\right)\right\}^{2}<1 .
$$

By Theorem $\mathrm{B}, f(x) \equiv \sin (x+\gamma)$ throughout an interval at one end of which $f(x)= \pm 1$. At this end point, say $x_{2}$, it follows by continuity that $\left\{f^{\prime \prime}\left(x_{2}\right)\right\}^{2}+\left\{f^{\prime}\left(x_{2}\right)\right\}^{2}=1$ and $f^{\prime}\left(x_{2}\right)=0$. Applying Theorem B to the function $f^{\prime}(x)$, we obtain $f^{\prime}(x) \equiv \cos (x+\gamma)$ over an interval extending a distance $\pi / 2$ on each side of $x_{2}$. At the end points of this interval $\left\{f^{\prime}(x)\right\}^{2}+\{f(x)\}^{2}=1$ and $f(x)=0$; so applying Theorem B to the function $f(x)$, we see that $f(x) \equiv \sin (x+\gamma)$ in an interval of length $2 \pi$. Clearly this process may be repeated to give a continuation of $f(x)$ for all values of $x$, and this proves the Theorem for $k=1$.

The problem for higher values of $k$ is easily reduced to the case $k=1$ just discussed. Suppose for some $x_{1}$ and $k$

$$
\left\{f^{(k)}\left(x_{1}\right)\right\}^{2}+\left\{f^{(k-1)}\left(x_{1}\right)\right\}^{2}=1, \quad 1<k<n .
$$

By application of Theorem B to the function $f^{(k-1)}(x)$ we see that there is a point where $f^{(k-1)}(x)= \pm 1$. At this point we have

$$
\left\{f^{(k-1)}(x)\right\}^{2}+\left\{f^{(k-2)}(x)\right\}^{2}=1, \quad f^{(k-2)}(x)=0 .
$$

Now, note that (2) is the same as (1) with $k$ replaced by $k-1$ and with the added condition that $f^{(k-2)}(x)$ is zero. By repeating this proc- 
ess a sufficient number of times we shall find that at some point $\left\{f^{\prime}(x)\right\}^{2}+\{f(x)\}^{2}=1$ and $f(x)=0$. This is an instance of the case already discussed, and the theorem is proved.

We are now able to give a proof of S. Bernstein's theorem on trigonometric polynomials, which may be stated in the following form: ${ }^{6}$

If $\phi(x)$ is a trigonometric polynomial of degree $N$ or less, $\phi(x)=\sum_{0}^{N}\left(a_{\nu} \cos \nu x+b_{\nu} \sin \nu x\right)$; and if $|\phi(x)| \leqq 1$ for all $x$, then $\left|\phi^{\prime}(x)\right|<N$ for all $x$, unless $\phi(x) \equiv \sin (N x+\alpha)$ for some constant $\alpha$.

Proof. Let $f(x)=\phi(x / \lambda)$ where $\lambda$ is some constant greater than $N$. Then as $n \rightarrow \infty, f^{(n)}(x) \rightarrow 0$ uniformly in $(-\infty, \infty)$; hence for sufficiently large $n,\left\{f^{(n)}(x)\right\}^{2}+\left\{f^{(n-1)}(x)\right\}^{2} \leqq 1$. Thus, by Theorem A we have $\left\{f^{(k)}(x)\right\}^{2}+\left\{f^{(k-1)}(x)\right\}^{2} \leqq 1$, or

$$
\left\{\frac{1}{\lambda^{k}} \phi^{(k)}(x)\right\}^{2}+\left\{\frac{1}{\lambda^{k-1}} \phi^{(k-1)}(x)\right\}^{2} \leqq 1, \quad k=1,2,3, \cdots .
$$

Considering any fixed $k$ and letting $\lambda \rightarrow N+0$, we obtain

$$
\left\{\frac{1}{N^{k}} \phi^{(k)}(x)\right\}^{2}+\left\{\frac{1}{N^{k-1}} \phi^{(k-1)}(x)\right\}^{2} \leqq 1, \quad k=1,2,3, \cdots .
$$

To obtain conditions of equality Theorem $\mathrm{C}$ is directly applicable.

Finally we give another simple inference from Theorem A.

If $f_{1}(x)$ and $f_{2}(x)$ satisfy the conditions of Theorem A for some integer $n$, then

$$
\left|\frac{d^{k}}{d x^{k}} f_{1}(x) f_{2}(x)\right| \leqq 2^{k-1}, \quad k=1,2,3, \cdots, n .
$$

Proof. By the Leibniz rule

$$
\frac{d^{p}}{d x^{p}}\left(f_{1}^{\prime}+i f_{1}\right)\left(f_{2}^{\prime}+i f_{2}\right)=\sum_{0}^{p} C_{p, \nu}\left(f_{1}^{(p+1)}+i f_{1}^{(\nu)}\right) \cdot\left(f_{2}^{(p+1-p)}+i f_{2}^{(p-v)}\right)
$$

where the constants $C_{p, \nu}$ are the binomial coefficients. Then by Theorem A

$$
\sum_{0}^{p} C_{p, \nu}\left|f_{1}^{(\nu+1)}+i f_{1}^{(\nu)}\right| \cdot\left|f_{2}^{(p+1-\nu)}+i f_{2}^{(p-\nu)}\right| \leqq \sum_{0}^{p} C_{p, \nu}=2^{p} .
$$

${ }^{6}$ M. Riesz, Jahresbericht der Deutschen Mathematiker-Vereinigung, vol. 23 (1914), pp. 354-368. C. J. de la Vallée Poussin, Comptes Rendus de l'Académie des Sciences, vol. 166 (1918), pp. 843-846. G. P6lya and G. Szegö, Aufgaben und Lehrsätze aus der Analysis, vol. 2, pp. 35, 90. 
To separate real and imaginary parts we may write

$$
\left(f_{1}^{\prime}+i f_{1}\right)\left(f_{2}^{\prime}+i f_{2}\right)=f_{1}^{\prime} f_{2}^{\prime}-f_{1} f_{2}+i \frac{d}{d x}\left(f_{1} f_{2}\right) .
$$

Differentiating this last expression $p$ times and taking absolute magnitudes, we obtain

$$
\left\{\frac{d^{p+1}}{d x^{p+1}}\left(f_{1} f_{2}\right)\right\}^{2}+\left\{\frac{d^{p}}{d x^{p}}\left(f_{1}^{\prime} f_{2}^{\prime}-f_{1} f_{2}\right)\right\}^{2} \leqq\left(2^{p}\right)^{2},
$$

which is more than we set out to prove. The functions $f_{1}(x)=\sin \left(x+\alpha_{1}\right)$ and $f_{2}(x)=\sin \left(x+\alpha_{2}\right)$ show that our constant is the "best possible."

Purdue University and

STANFORD UNIVERSITY

\title{
ON THE CARATHEODORY CONDITION FOR UNILATERAL VARIATIONS ${ }^{1}$
}

\author{
JULIAN D. MANCILL
}

The two formulations and proofs of the Carathéodory condition in the calculus of variations given by Graves ${ }^{2}$ do not necessarily apply to the case when the minimizing curve may have arcs in common with the boundary of the region of admissible variations. ${ }^{3}$ The purpose of this note is to show how his first formulation and proof can be modified so as to be applicable to unilateral (one-sided) variations in the plane.

An admissible curve

$$
E_{0}: \quad x^{\alpha}=x^{\alpha}(t)
$$$$
t_{0} \leqq t \leqq T, \alpha=1,2, \cdots, n
$$

which minimizes the integral

$$
J=\int_{t_{0}}^{T} F\left(x_{1}, \cdots, x_{n}, x_{1}^{\prime}, \cdots, x_{n}^{\prime}\right) d t \equiv \int_{t_{0}}^{T} F\left(x, x^{\prime}\right) d t
$$

in the class of all admissible curves joining two fixed points $x_{0}$ and $X$ in space of $n$ dimensions ( $n>1$ ), must satisfy certain well known con-

${ }_{1}^{1}$ Presented to the Society, December 29, 1939.

2 Discontinuous solutions in space problems of the calculus of variations, American Journal of Mathematics, vol. 52 (1930), pp. 13-19.

${ }^{3} \mathrm{Cf}$. Mancill, The minimum of a definite integral with respect to unilateral variations, Contributions to the Calculus of Variations, 1933-37, University of Chicago, 1937, p. 121, condition $C_{3}$. 